\title{
Democratic Approximation of Lexicographic Preference Models
}

Fusun Yaman

FUSUN@CS.UMBC.EDU

University of Maryland Baltimore County, Computer Science and Electrical Eng. Department, Baltimore, MD 21250 USA

Thomas J. Walsh

Michael L. Littman

THOMASWA@CS.RUTGERS.EDU

MLITTMAN@CS.RUTGERS.EDU

Rutgers University, Department of Computer Science, Piscataway, NJ 08854 USA

Marie desJardins

MARIEDJ@CS.UMBC.EDU

University of Maryland Baltimore County, Computer Science and Electrical Eng. Department, Baltimore, MD 21250 USA

\begin{abstract}
Previous algorithms for learning lexicographic preference models (LPMs) produce a "best guess" LPM that is consistent with the observations. Our approach is more democratic: we do not commit to a single LPM. Instead, we approximate the target using the votes of a collection of consistent LPMs. We present two variations of this method-variable voting and model voting - and empirically show that these democratic algorithms outperform the existing methods. We also introduce an intuitive yet powerful learning bias to prune some of the possible LPMs. We demonstrate how this learning bias can be used with variable and model voting and show that the learning bias improves the learning curve significantly, especially when the number of observations is small.
\end{abstract}

\section{Introduction}

Lexicographic preference models (LPMs) are one of the simplest preference representations. An LPM defines an order of importance on the variables that describe the objects in a domain and uses this order to make preference decisions. For example, the meal preference of a vegetarian with a weak stomach could be represented by an LPM such that a vegetarian dish is always preferred over a nonvegetarian dish, and among vegetarian or non-vegetarian items, mild dishes are preferred to spicy ones. Previous work on learning LPMs from a set of preference observations has been limited to autocratic approaches: one of

Appearing in Proceedings of the $25^{\text {th }}$ International Conference on Machine Learning, Helsinki, Finland, 2008. Copyright 2008 by the author(s)/owner(s). many possible LPMs is picked heuristically and used for future decisions. However, it is highly likely that autocratic methods will produce poor approximations of the target when there are few observations.

In this paper, we present a democratic approach to LPM learning, which does not commit to a single LPM. Instead, we approximate a target preference using the votes of a collection of consistent LPMs. We present two variations of this method: variable voting and model voting. Variable voting operates on the variable level and samples the consistent LPMs implicitly. The learning algorithm based on variable voting learns a partial order on the variables where all linearizations correspond to an LPM consistent with the observations. Model voting explicitly samples the consistent LPMs and employs weighted voting where the weights are computed using Bayesian priors. The additional complexity of voting-based algorithms is tolerable: both algorithms have low-order polynomial time complexity. Our experiments show that these democratic algorithms outperform more than half of the LPMs that can be produced by an autocratic algorithm, greatly increasing the chance of a positive outcome.

To further improve the performance of the learning algorithms when the number of observations is small, we introduce an intuitive yet powerful learning bias. The bias defines equivalence classes on the variables, indicating the most important set of variables, the second most important set, and so on. We demonstrate how this learning bias can be used with variable and model voting and show that the learning bias improves the learning curve significantly on appropriate problems, especially when the number of observations is small.

In the rest of the paper, we give some background on LPMs, then introduce our voting-based methods. We then introduce the learning bias and show how we can generalize the 
voting methods to utilize such a bias. Finally, we present the results of our experiments, followed by related work and concluding remarks.

\section{Lexicographic Decision Models}

In this section, we briefly introduce the lexicographic preference model (LPM) and summarize previous results on learning LPMs. Before going into the definition of an LPM, we state that we only consider binary variables whose domain is $\{0,1\} .{ }^{1}$ Like others before us, we assume that the preferred value of each variable is known. Without loss of generality, we will assume that 1 is always preferred to 0 .

Given a set of variables, $X=\left\{X_{1} \ldots X_{n}\right\}$, an object $A$ over $X$ is a vector of the form $\left[x_{1}, \ldots, x_{n}\right]$. We use the notation $A\left(X_{i}\right)$ to refer the value of $X_{i}$ in the object $A$. A lexicographic preference model $\mathcal{L}$ on $X$ is a total order on a subset $R$ of $X$. We denote this total order with $\sqsubset_{\mathcal{L}}$. Any variable in $R$ is relevant with respect to $\mathcal{L}$; similarly, any variable in $X-R$ is irrelevant with respect to $\mathcal{L}$. If $A$ and $B$ are two objects, then the preferred object given $\mathcal{L}$ is determined as follows:

- Find the smallest variable $X^{*}$ in $\sqsubset_{\mathcal{L}}$ such that $X^{*}$ has different values in $A$ and $B$. The object that has the value 1 for $X^{*}$ is the most preferred.

- If all relevant variables in $\mathcal{L}$ have the same value in $A$ and $B$, then the objects are equally preferred (a tie).

Example 1 Suppose $X_{1}<X_{2}<X_{3}$ is the total order defined by an $L P M \mathcal{L}$, and consider objects $A=[1,0,1,1]$, $B=[0,1,0,0], C=[0,0,1,1]$ and $D=[0,0,1,0] . A$ is preferred over $B$ because $A\left(X_{1}\right)=1$, and $X_{1}$ is the most important variable in $\mathcal{L}$. $B$ is preferred over $C$ because $B\left(X_{2}\right)=1$ and both objects have the same value for $X_{1}$. Finally, $C$ and $D$ are equally preferred because they have the same values for the relevant variables.

An observation $o=(A, B)$ is an ordered pair of objects, connoting that $A$ is preferred to $B$. In many practical applications, however, preference observations are gathered from demonstration of an expert who breaks ties arbitrarily. Thus, for some observations, $A$ and $B$ may actually be tied. An LPM $\mathcal{L}$ is consistent with an observation $(A, B)$ iff $\mathcal{L}$ implies that $A$ is preferred to $B$ or that $A$ and $B$ are equally preferred.

The problem of learning an LPM is defined as follows. Given a set of observations, find an LPM $\mathcal{L}$ that is consistent with the observations. Previous work on learning LPMs was limited to the case where all variables are relevant. This assumption entails that, in every observation

\footnotetext{
${ }^{1}$ The representation can easily be generalized to monotonic preferences with ordinal variables such that 1 corresponds to a preference on the increasing order and 0 on decreasing order.
}

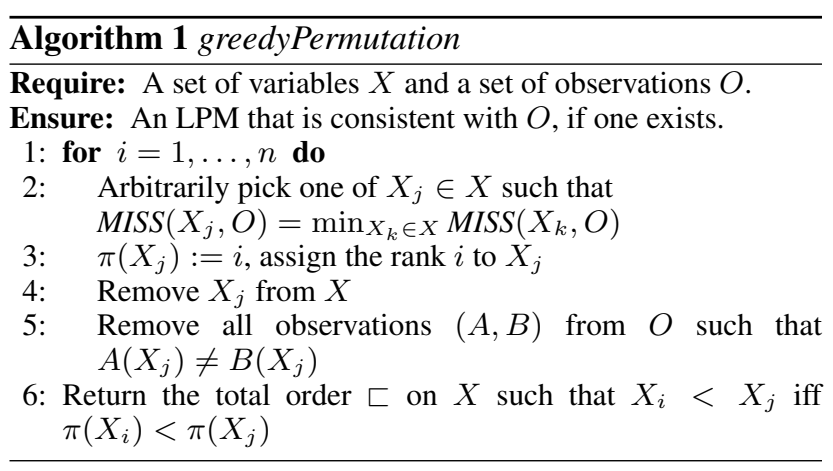

( $A, B), A$ is strictly preferred to $B$, since ties can only happen when there are irrelevant attributes.

Schmitt and Martignon (2006) proposed a greedy algorithm that is guaranteed to find one of the LPMs that is consistent with the observations if one exists. They have also shown that for the noisy data case, finding an LPM that does not violate more than a constant number of the observations is NP-complete. Algorithm 1 is Schmitt and Martignon's greedy variable-permutation algorithm, which we use as a performance baseline. The algorithm refers to a function $\operatorname{MISS}\left(X_{i}, O\right)$, which is defined as $\mid\{(A, B) \in O$ : $\left.A\left(X_{i}\right)<B\left(X_{i}\right)\right\} \mid$; that is, the number of observations violated in $O$ if the most important variable is selected as $X_{i}$. Basically, the algorithm greedily constructs a total order by choosing the variable at each step that causes the minimum number of inconsistencies with the observations. If multiple variables have the same minimum, then one of them is chosen arbitrarily. The algorithm runs in polynomial time, specifically $O\left(n^{2} m\right)$, where $n$ is the number of variables and $m$ is the number of observations.

Dombi et al. (2007) have shown that if there are $n$ variables, all of which are relevant, then $O(n \log n)$ queries to an oracle suffice to learn an LPM. Furthermore, it is possible to learn any LPM with $O\left(n^{2}\right)$ observations if all pairs differ in only two variables. They proposed an algorithm that can find the unique LPM induced by the observations. In case of noise due to irrelevant attributes the algorithm does not return an answer.

In this paper, we investigate the following problem: Given a set of observations with no noise, but possibly with arbitrarily broken ties, find a rule for predicting preferences that agrees with the target LPM that produced the observations.

\section{Voting Algorithms}

We propose a democratic approach for approximating the target LPM that produced a set of observations. Instead of finding just one of the consistent LPMs, it reasons with a collection of LPMs that are consistent with the observations. Given two objects, such an approach prefers the one 
that a majority of its models prefer. A naive implementation of a voting algorithm would enumerate all LPMs that are consistent with a set of observations. However, since the number of models consistent with a set of observations can be exponential, the naive implementation is infeasible.

In this section, we describe two methods-variable voting and model voting - that sample the set of consistent LPMs and use voting to predict the preferred object. Unlike existing algorithms that learn LPMs, these methods do not require all variables to be relevant or observations to be tiefree. The following subsections explain the variable voting and model voting methods and summarize some of our theoretical results.

\subsection{Variable Voting}

Variable voting uses a generalization of the LPM representation. Instead of a total order on the variables, variable voting reasons with a partial order $(\preceq)$ to find the preferred object in a given pair. Among the variables that are different in both objects, the ones that have the smallest rank (and are hence the most salient) in the partial order vote to choose the preferred object. The object that has the most " 1 " values for the voting variables is declared to be the preferred one. If the votes are equal, then the objects are equally preferred.

Definition 1 (Variable Voting) Suppose $X$ is a set of variables and $\preceq$ is a partial order on $X$. Given two objects, $A$ and $B$, the variable voting process with respect to $\preceq$ for determining which of the two objects is preferred is:

- Define $D$, the set of variables that differ in $A$ and $B$.

- Define $D^{*}$, the set of variables in $D$ that have the smallest rank among $D$ with respect to $\preceq$.

- Define $N_{A}$ as the number of variables in $D^{*}$ that favor $A$ (i.e., that have value 1 in $A$ and 0 in $B$ ) and $N_{B}$, as the number of variables in $D^{*}$ that favor $B$.

- If $N_{A}>N_{B}$, then $A$ is preferred. If $N_{A}<N_{B}$, then $B$ is preferred. Otherwise, they are equally preferred.

Example 2 Suppose $\preceq$ is the partial order $\left\{X_{2}, X_{3}\right\}<$ $\left\{X_{1}\right\}<\left\{X_{4}, X_{5}\right\}$. Consider objects $A=[0,1,1,0,0]$ and $B=[0,0,1,0,1] . D$ is $\left\{X_{2}, X_{5}\right\} . D^{*}$ is $\left\{X_{2}\right\}$ because $X_{2}$ is the smallest ranking variable in $D$ with respect to $\preceq$. $X_{2}$ favors $A$ because $A\left(X_{2}\right)=1$. Thus, variable voting with $\preceq$ prefers $A$ over $B$.

Algorithm 2 presents the algorithm learnVariableRank, which learns a partial order $\preceq$ on the variables from a set of observations such that variable voting with respect to $\preceq$ will correctly predict the preferred objects in the observations. Specifically, it finds partial orders that define equivalence classes on the set of variables. The algorithm

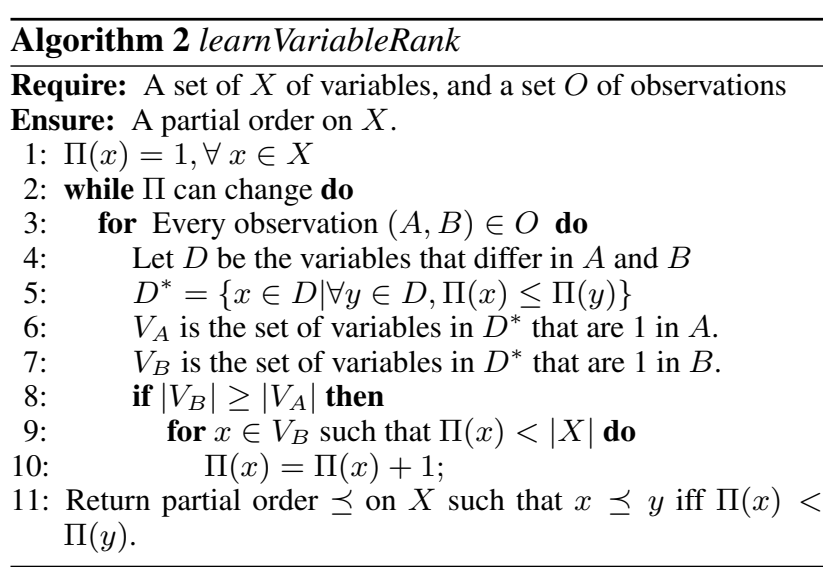

Table 1. The rank of the variables after each iteration of the forloop in line 3 of the algorithm learnVariableRank.

\begin{tabular}{|l|l|l|l|l|l|}
\hline Observations & $X_{1}$ & $X_{2}$ & $X_{3}$ & $X_{4}$ & $X_{5}$ \\
\hline Initially & 1 & 1 & 1 & 1 & 1 \\
\hline$[0,1,1,0,0],[1,1,0,1,1]$ & 2 & 1 & 1 & 2 & 2 \\
\hline$[0,1,1,0,1],[1,0,0,1,0]$ & 2 & 1 & 1 & 2 & 2 \\
\hline$[1,0,1,0,0],[0,0,1,1,1]$ & 2 & 1 & 1 & 3 & 3 \\
\hline
\end{tabular}

maintains the minimum possible rank for every variable that does not violate an observation with respect to variable voting. Initially, all variables are considered equally important (rank of 1). The algorithm loops over the set of observations until the ranks converge. At every iteration and for every pair, variable voting predicts a winner. If it is correct, then the ranks stay the same. Otherwise, the ranks of the variables that voted for the wrong object are incremented, thus reducing their importance ${ }^{2}$. Finally, the algorithm builds a partial order $\preceq$ based on the ranks such that $x \preceq y$ if and only if $x$ has a lower rank than $y$.

Example 3 Suppose $X=\left\{X_{1}, X_{2}, X_{3}, X_{4}, X_{5}\right\}$ and $O$ consists of $([0,1,1,0,0],[1,1,0,1,1]),([0,1,1,0,1]$, $[1,0,0,1,0])$ and $([1,0,1,0,0],[0,0,1,1,1])$. Table 1 illustrates the ranks of every variable in $X$ after each iteration of the for-loop in line 3 of the algorithm learnVariableRank. The ranks of the variables stay the same during the second iteration of the while-loop, thus, the loop terminates. The partial order $\preceq$ based on ranks of the variables is the same as the order given in Example 2.

We next summarize our theoretical results about the algorithm learnVariableRank.

Correctness: Suppose $\preceq$ is a partial order returned by learn VariableRank $(X, O)$. It can be shown that any LPM $\mathcal{L}$ such that $\sqsubset_{\mathcal{L}}$ is a topological sort of $\preceq$ is consistent with

\footnotetext{
${ }^{2}$ In our empirical results, we also update the ranks when the prediction was correct but not unanimous. This produces a heuristic speed-up without detracting from the worst case guarantees.
} 
O. Furthermore, learn VariableRank never increments the ranks of the relevant variables beyond their actual rank in the target LPM. The ranks of the irrelevant variables can be incremented as far as the number of variables.

Convergence: learnVariableRank has a mistake-bound of $O\left(n^{2}\right)$, where $n$ is the number of variables, because each mistake increases the sum of the potential ranks by at least 1 and the sum of the ranks the target LPM induces is $O\left(n^{2}\right)$. This bound guarantees that given enough observations (as described in the background section), learnVariableRank will converge to a partial order $\preceq$ such that every topological sort of $\preceq$ has the same prefix as the total order induced by the target LPM. If all variables are relevant, then $\preceq$ will converge to the total order induced by the target LPM.

Complexity: A very loose upper bound on the time complexity of learnVariableRank is $O\left(n^{3} m\right)$, where $n$ is the number of variables and $m$ is the number of observations. This bound holds because the while-loop on line 2 runs at most $O\left(n^{2}\right)$ times and the for-loop in line 3, runs for $m$ observations. The time complexity of one iteration of the forloop is $O(n)$; therefore, the overall complexity is $O\left(n^{3} m\right)$. We leave the investigation of tighter bounds and the average case analysis for future work.

\subsection{Model Voting}

The second method we present employs a Bayesian approach. This method randomly generates a sample set, $S$, of distinct LPMs, that are consistent with the observations. When a pair of objects is presented, the preferred one is predicted using weighted voting. That is, each $\mathcal{L} \in S$ casts a vote for the object it prefers, and this vote is weighted according to its posterior probability $P(\mathcal{L} \mid S)$.

Definition 2 (Model Voting) Let $U$ be the set of all LPMs, $O$ be a set of observations, and $S \subset U$ be a set of LPMs that are consistent with $O$. Given two objects $A$ and $B$, model voting prefers $A$ over $B$ with respect to $S$ if

$$
\sum_{\mathcal{L} \in U} P(\mathcal{L} \mid S) V_{(A>B)}^{\mathcal{L}}>\sum_{\mathcal{L} \in U} P(\mathcal{L} \mid S) V_{(B>A)}^{\mathcal{L}},
$$

where $V_{(A>B)}^{\mathcal{L}}$ is 1 if $A$ is preferred with respect to $\mathcal{L}$, and 0 otherwise. $V_{(B>A)}^{\mathcal{L}}$ is defined analogously. $P(\mathcal{L} \mid S)$ is the posterior probability of $\mathcal{L}$ being the target LPM given $S$, calculated as discussed below.

We first assume that all LPMs are equally likely a priori. In this case, given a sample $S$ of size $k$, the posterior probability of an $\operatorname{LPM} \mathcal{L}$ will be $1 / k$ if and only if $\mathcal{L} \in S$, and 0 otherwise. Note that if $S$ is maximal this case degenerates into the naive voting algorithm. However, it is generally not

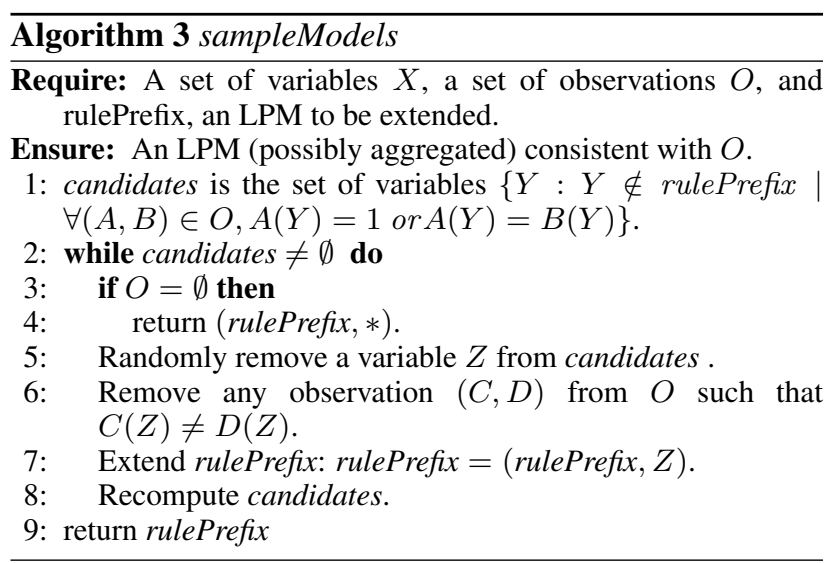

feasible to have all consistent LPMs-in practice, the sample has to be small enough to be feasible and large enough to be representative.

In constructing $S$, we exploit the fact that many consistent LPMs share prefixes in the total order that they define on the variables. We wish to discover and compactly represent such LPMs. To this end, we introduce the idea of $a g$ gregated LPMs. An aggregated LPM, $\left(X_{1}, X_{2} \ldots, X_{k}, *\right)$, represents a set of LPMs that define a total order with the prefix $X_{1}<X_{2}<\ldots<X_{k}$. Intuitively, an aggregated LPM states that any possible completion of the prefix is consistent with the observations. The algorithm sampleModels in Algorithm 3 implements a "smart sampling" approach by constructing an LPM that is consistent with the given observations, returning an aggregated LPM when possible. We start with an arbitrary consistent LPM (such as the empty set, which is always consistent) and add more variable orderings extending the input LPM. We first identify the variables that can be used in extending the prefixthat is, all variables $X_{i}$ such that in every observation, either $X_{i}$ is 1 in the preferred object or is the same in both objects. We then select one of those variables randomly and extend the prefix. Finally, we remove the observations that are explained with this selection and continue with the rest of the observations. If at any point, no observations remain, then we return the aggregated form of the prefix, since every completion of the prefix will be consistent with the null observation. Running sampleModels several times and eliminating duplicates will produce a set of (possibly aggregated) LPMs.

Example 4 Consider the same set of observations $O$ as in Example 3. Then, the LPMs that are consistent with $O$ are as follows: (), $\left(X_{2}\right),\left(X_{2}, X_{3}\right),\left(X_{2}, X_{3}, X_{1}, *\right),\left(X_{3}\right)$, $\left(X_{3}, X_{1}, *\right),\left(X_{3}, X_{2}\right)$ and $\left(X_{3}, X_{2}, X_{1}, *\right)$. To illustrate the set of LPMs that an aggregate LPM represents, consider $\left(X_{2}, X_{3}, X_{1}, *\right)$, which has a total of 5 extensions: $\left(X_{2}, X_{3}, X_{1}\right), \quad\left(X_{2}, X_{3}, X_{1}, X_{4}\right), \quad\left(X_{2}, X_{3}, X_{1}, X_{5}\right)$, $\left(X_{2}, X_{3}, X_{1}, X_{4}, X_{5}\right), \quad\left(X_{2}, X_{3}, X_{1}, X_{5}, X_{4}\right)$. Every 
time the algorithm sampleModels runs, it will randomly generate one of the aggregated LPMs: $\left(X_{2}, X_{3}, X_{1}, *\right)$, $\left(X_{3}, X_{1}, *\right)$, or $\left(X_{3}, X_{2}, X_{1}, *\right)$. Note that the shorter models that are not produced by sampleModels are all sub-prefixes of the aggregated LPMs and it is easy to modify sampleModels to return those models as well.

An aggregate LPM in a sample saves us from enumerating all possible extensions of a prefix, but it also introduces complications in computing the weights (posteriors) of the LPMs, as well as their votes. For example, when comparing two objects $A$ and $B$, some extensions of an aggregate LPM might vote for $A$ and some for $B$. Thus, we need to find the total number of LPMs that an aggregate LPM represents and determine what proportion of them favor $A$ over $B$ (or vice versa), without enumerating all extensions. Suppose there are $n$ variables and $\mathcal{L}$ is an aggregated LPM with a prefix of length $k$. Then, the number of extensions of $\mathcal{L}$ is denoted by $F_{\mathcal{L}}$ and is equal to $f_{n-k}$, where $f_{m}$ is defined to be:

$$
f_{m}=\sum_{i=0}^{m}\left(\begin{array}{c}
m \\
i
\end{array}\right) \times i !=\sum_{i=0}^{m} \frac{(m) !}{(m-i) !} .
$$

Intuitively, $f_{m}$ counts every possible permutation with at most $m$ items. Note that $f_{m}$ can be computed efficiently and that the number of all possible LPMs when there are $n$ variables is given by $f_{n}$.

Consider a pair of objects $A$ and $B$. We wish to determine how many extensions of an aggregate LPM $\mathcal{L}=$ $\left(X_{1}, X_{2}, \ldots, X_{k}, *\right)$ would vote for one of the objects. We will call the variables $X_{1} \ldots X_{k}$ the prefix variables. If $A$ and $B$ have different values for at least one prefix variable, then all extensions will vote in accordance with the smallest such variable. Suppose all prefix variables are tied and $m$ is the set of all non-prefix variables. Then, $m$ is composed of three disjoint sets $a, b$, and $w$, such that $a$ is the set of variables that favor $A, b$ is the set of variables that favor $B$, and $w$ is the set of variables that are neutral (that is, that have the same value in $A$ and $B$ ).

An extension $\mathcal{L}^{\prime}$ of $\mathcal{L}$ will produce a tie iff all variables in $a$ and $b$ are irrelevant in $\mathcal{L}^{\prime}$. The number of such extensions is $f_{|w|}$. The number of extensions that favor $A$ over $B$ is directly proportional to $|a| /(|a|+|b|)$. The number of extensions of $\mathcal{L}$ that will vote for $A$ over $B$ is denoted by $N_{A>B}^{\mathcal{L}}$, which is given by:

$$
N_{A>B}^{\mathcal{L}}=\frac{|a|}{|b|+|a|} \times\left(f_{m}-f_{|w|}\right) .
$$

The number of extensions of $\mathcal{L}$ that will vote for $B$ over $A$ is computed similarly. Note that the computation of $N_{A>B}^{\mathcal{L}}$, $N_{B>A}^{\mathcal{L}}$, and $F_{\mathcal{L}}$ can be done in linear time by caching the recurrent values.
Table 2. The posterior probabilities and number of votes of all LPMs in Example 5.

\begin{tabular}{|l|l|l|l|l|}
\hline LPMs & $P\left(L \mid S_{1}\right)$ & $P\left(L \mid S_{2}\right)$ & $N_{A>B}^{\mathcal{L}}$ & $N_{B>A}^{\mathcal{L}}$ \\
\hline() & $1 / 31$ & 0 & 0 & 0 \\
\hline$\left(X_{2}\right)$ & $1 / 31$ & 0 & 1 & 0 \\
\hline$\left(X_{2}, X_{3}\right)$ & $1 / 31$ & 0 & 1 & 0 \\
\hline$\left(X_{2}, X_{3}, X_{1}, *\right)$ & $5 / 31$ & $5 / 26$ & 5 & 0 \\
\hline$\left(X_{3}\right)$ & $1 / 31$ & 0 & 0 & 0 \\
\hline$\left(X_{3}, X_{1}, *\right)$ & $16 / 31$ & $16 / 26$ & 7 & 7 \\
\hline$\left(X_{3}, X_{2}\right)$ & $1 / 31$ & 0 & 1 & 0 \\
\hline$\left(X_{3}, X_{2}, X_{1}, *\right)$ & $5 / 31$ & $5 / 26$ & 5 & 0 \\
\hline
\end{tabular}

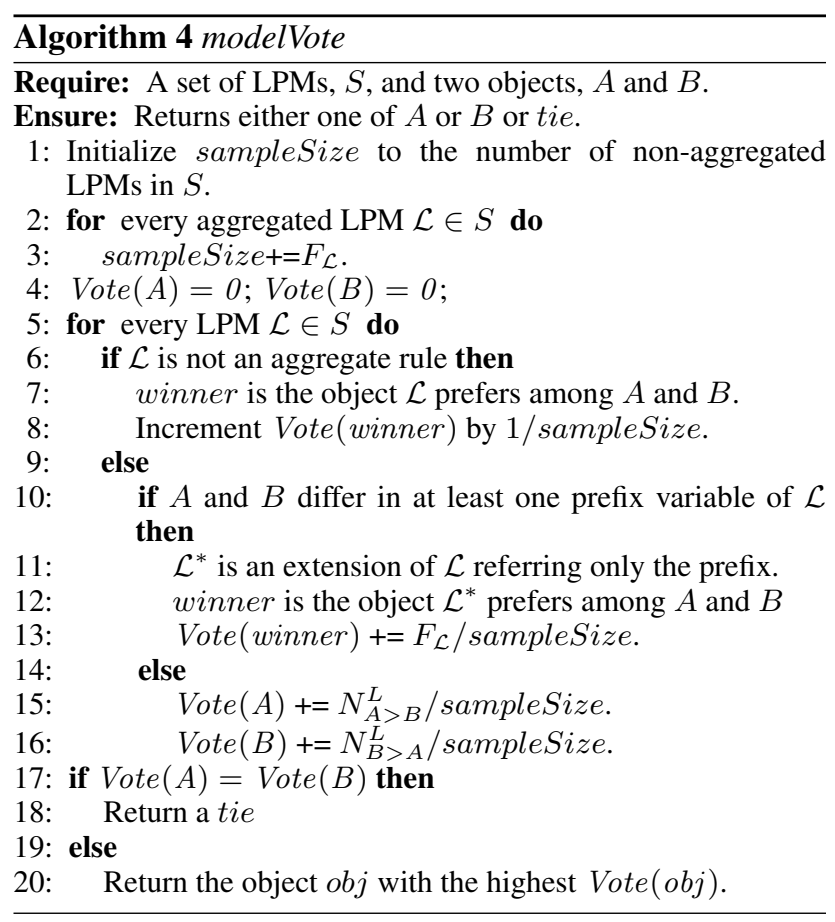

Example 5 Suppose $X$ and $O$ are as defined in Example 3. The first column of Table 2 lists all LPMs that are consistent with $O$. The second column gives the posterior probabilities of these models given the sample $S_{1}$, which is the set of all consistent LPMs. The third column is the posterior probability of the models given the sample $S_{2}=$ $\left\{\left(X_{2}, X_{3}, X_{1}, *\right),\left(X_{3}, X_{1}, *\right),\left(X_{3}, X_{2}, X_{1}, *\right)\right\}$. Given two objects $A=[0,1,1,0,0]$ and $B=[0,0,1,0,1]$, the number of votes for each object based on each LPM is given in the last two columns. Note that the total number of votes for $A$ and $B$ does not add up to the total number of extensions of $\left(X_{3}, X_{1}, *\right)$ because two of its extensions$\left(X_{3}, X_{1}\right)$ and $\left(X_{3}, X_{1}, X_{4}\right)$-prefer $A$ and $B$ equally.

Algorithm 4 describes modelVote, which takes a sample of consistent LPMs and a pair of objects as input, and predicts the preferred object using the weighted votes of the LPMs in the sample. 
Returning to Example 5, the reader can verify that model voting will prefer $A$ over $B$. Next, we present our theoretical results on the sampleModels and modelVote algorithms.

Complexity: The time complexity of sampleModels is bounded by $O\left(n^{2} m\right)$, where $n$ is the number of variables and $m$ is the number of observations: the while-loop in line 2 runs at most $n$ times; at each iteration, we have to process every observation, each time performing computations in $O(n)$ time. If we call sampleModels $s$ times, then the total complexity of sampling is $O\left(s n^{2} m\right)$. For constant $s$, this bound is still polynomial. Similarly, the complexity of modelVote is $O(s n)$ because it considers each of the $s$ rules in the sample, counting the votes of each rule, which can be done in $O(n)$ time.

Comparison to variable voting: The set of LPMs that is sampled via learnVariableRank is a subset of the LPMs that sampleModels can produce. The running example in the paper demonstrates that sampleModels can generate the LPM $\left(X_{3}, X_{1}, *\right)$; however, none of its extensions is consistent with the partial order learnVariableRank returns.

\section{Introducing Bias}

In general, when there are not many training examples for a learning algorithm, the space of consistent LPMs is large. In this case, it is not possible to find a good approximation of the target model. To overcome this problem, we can introduce bias (domain knowledge), indicating that certain solutions should be favored over the others. In this section, we propose a bias in the form of equivalence classes over the set of attributes. These equivalence classes indicate the set of most important attributes, second most important attributes, and so on. For example, when buying a used car, most people consider the most important attributes of a car to be the mileage, the year, and the make of the car. The second most important set of attributes is the color, number of doors, and body type. Finally, perhaps the least important properties are the interior color and the wheel covers. We now formally define a learning bias and what it means for an LPM to be consistent with a learning bias.

Definition 3 (Learning Bias) A learning bias $\mathcal{B}$ for learning a lexicographic preference model on a set of variables $X$ is a total order on a partition of $X . \mathcal{B}$ has the form $E_{1}<E_{2}<\ldots<E_{k}$, where $\cup_{i} E_{i}=X$. Intuitively, $B$ defines a partial order on $X$ such that for any two variables $x \in E_{i}$ and $y \in E_{j}, x<y$ iff $E_{i}<E_{j}$. We denote this partial order by $\preceq_{B}$.

Definition 4 Suppose that $X=\left\{X_{1}, \ldots X_{n}\right\}$ is a set of variables, $\mathcal{B}$ a learning bias, and $\mathcal{L}$ an LPM. $\mathcal{L}$ is consistent with $\mathcal{B}$ iff the total order $\sqsubset_{\mathcal{L}}$ is consistent with the partial $\operatorname{order} \preceq \mathcal{B}$.

Intuitively, an LPM that is consistent with a learning bias respects the variable orderings induced by the learning bias. The learning bias prunes the space of possible LPMs. The size of the partition determines the strength of the bias; for example, if there is a single variable per set, then the bias defines a specific LPM. In general, the number of LPMs that is consistent with a learning bias of the form $E_{1}<E_{2}<\ldots<E_{k}$ can be computed with the following recursive formula:

$$
G\left(\left[e_{1}, \ldots e_{k},\right]\right)=f_{e_{1}}+e_{1} ! \times\left(G\left(\left[e_{2}, \ldots e_{k}\right]\right)-1\right),
$$

where $e_{i}=\left|E_{i}\right|$ and the base case for the recursion is $G([])=1$. The first term in the formula counts the number of possible LPMs using only the variables in $E_{1}$, which are the most important variables. The definition of consistency entails that a variable can appear in $\sqsubset_{\mathcal{L}}$ iff all of the more important variables are already in $\sqsubset_{\mathcal{L}}$, hence the term $e_{1}$ !. Note that the recursion on $G$ is limited to the number of sets in the partition, which is bounded by the number of variables; therefore, it can also be computed in linear time by caching precomputed values of $f$.

To illustrate the power of a learning bias, consider a learning problem with nine variables. Without a bias, the total number of LPMs is 905,970. If a learning bias partitions the variables into three sets, each with three elements, then the number of LPMs consistent with the bias is only 646 . A bias with four sets, where the first set has three variables and the rest have two, limits the number to 190.

We can easily generalize the learnVariableRank algorithm to utilize the learning bias, by changing only the first line of learnVariableRank which initializes the ranks of the variables. Given a bias of the form $S_{1}<\ldots<S_{k}$, the generalized algorithm assigns the rank 1 (most important rank) to the variables in $S_{1}$, rank $\left|S_{1}\right|+1$ to those in $S_{2}$, and so forth. This initialization ensures that an observation $(A, B)$ is used for learning the order of variables in a class $S_{i}$ only when $A$ and $B$ have the same values for all variables in classes $S_{1} \ldots S_{i-1}$ and have different values for at least one variable in $S_{i}$.

The algorithm modelVote can also be generalized to use a learning bias $\mathcal{B}$. In the sample generation phase, we use sampleModels as presented earlier, and then eliminate all rules whose prefixes are not consistent with the bias. Note that even if the prefix of an aggregated LPM $\mathcal{L}$ is consistent with a bias, this may not be the case for every extension of $\mathcal{L}$. Thus, in the algorithm modelVote, we need to change any references to $F_{\mathcal{L}}$ and $N_{A<B}^{\mathcal{L}}\left(\right.$ or $\left.N_{B<A}^{\mathcal{L}}\right)$ with $F_{\mathcal{L}}^{\mathcal{B}}$ and $N_{A<B}^{\mathcal{L}, \mathcal{B}}$ (or $N_{B<A}^{\mathcal{L}, \mathcal{B}}$ ), respectively, where:

- $F_{\mathcal{L}}^{\mathcal{B}}$ is the number of extensions of $\mathcal{L}$ that are consistent with $\mathcal{B}$, and 
- $N_{A<B}^{\mathcal{L}, \mathcal{B}}$ is the number of extensions of $\mathcal{L}$ that are consistent with $\mathcal{B}$ and prefer $A$. $\left(N_{B<A}^{\mathcal{L}, \mathcal{B}}\right.$ is similar. $)$

Suppose that $\mathcal{B}$ is a learning bias $E_{1}<\ldots<E_{m}$. Let $Y$ denote the prefix variables of an aggregate LPM $\mathcal{L}$ and $E_{k}$ be the first set such that at least one variable in $E_{k}$ is not in $Y$. Then, $F_{\mathcal{L}}^{\mathcal{B}}=G\left(\left[\left|E_{k}-Y\right|,\left|E_{k+1}-Y\right|, \ldots\left|E_{m}-Y\right|\right]\right)$.

When counting the number of extensions of $\mathcal{L}$ that are consistent with $\mathcal{B}$ and prefer $A$, we again need to examine the case where the prefix variables equally prefer the objects. Suppose $Y$ is as defined as above and $D_{i}$ denotes the set difference between $E_{i}$ and $Y$. Let $D_{j}$ be the first non-empty set and $D_{k}$ be the first set such that at least one variable in $D_{k}$ has different values in the two objects. Obviously, only the variables in $D_{k}$ will influence the prediction of the preferred object. If

- $d_{i}=\left|D_{i}\right|$, the cardinality of $D_{i}$, and

- $a$ is the set of variables in $D_{k}$ that favor $A, b$ is the set of variables in $D_{k}$ that favor $B$, and $w$ is the set of variables in $D_{k}$ that are neutral,

then $N_{A>B}^{\mathcal{L}, \mathcal{B}}$, the number of extensions of $\mathcal{L}$ that are consistent with $\mathcal{B}$ and prefer $A$, can be computed as follows:

$$
N_{A>B}^{\mathcal{L}, \mathcal{B}}=\frac{|a|}{|a|+|b|} \times\left(F_{\mathcal{L}}^{\mathcal{B}}-G\left(\left[d_{j} \ldots d_{k-1},|w|\right]\right)\right) .
$$

\section{Experiments}

In this section, we explain our experimental methodology and discuss the results of our empirical evaluations. We define the prediction performance of an algorithm $P$ with respect to a set of test observations $T$ as:

$$
\text { performance }(P, T)=\frac{\operatorname{Correct}(P, T)+0.5 \times \operatorname{Tie}(P, T)}{|T|}
$$

where $\operatorname{Correct}(P, T)$ is the number of observations in $T$ that are predicted correctly by $P$ and $\operatorname{Tie}(P, T)$ is the number of observations in $T$ that $P$ predicted as a tie. Note that an LPM returned by greedyPermutation never returns a tie. In contrast, variable voting with respect to a partial order in which every variable is equally important will only return ties, so the overall performance will be 0.5 , which is no better than randomly selecting the preferred objects. We will use $M V, V V$, and $G$ to denote the model voting, variable voting, and the greedy approximations of an LPM.

Given sets of training and test observations, $(O, T)$, we measure the average and worst performances of $V V, M V$ and $G$. When combined with learnVariableRank, $V V$ is a deterministic algorithm, so the average and worst performances of $V V$ are the same. However, this is not the case

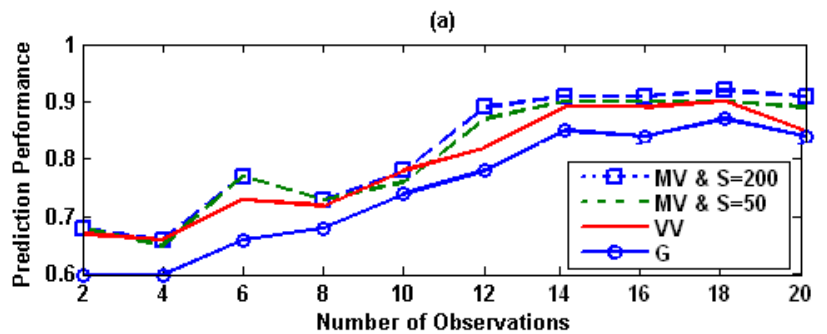

(b)

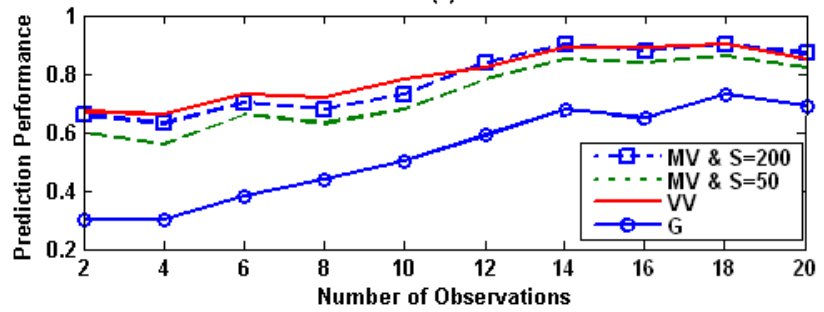

Figure 1. The average and worst prediction performance of the greedy algorithm, variable voting and model voting.

for $M V$ with sampling, because sampleModels is randomized. Even for the same training and test data $(O, T)$, the performance of $M V$ can vary. To mitigate this, we ran $M V 10$ times for each $(O, T)$ pair, and called sampleModels $S$ times on each run (thus the sample size is at most $\mathrm{S}$ ), recording the average and worst of its performance. The greedy algorithm $G$ is also randomized (in line 2, one variable is picked arbitrarily), so we ran $G 200$ times for every $(O, T)$, recording its average and worst performance.

For our experiments, the control variables are $R$, the number of relevant variables in the target LPM; $I$, the number of irrelevant variables; $N_{O}$, the number of training observations; and $N_{T}$, the number of test observations. For MV experiments we used sample sizes $(S)$ of 50 and 200. Larger sample sizes (e.g. 800) slightly improved performance, but are omitted for space. For fixed values of $R$ and $I$, an LPM $\mathcal{L}$ is randomly generated. (If a bias $B$ is given, then $\mathcal{L}$ is also consistent with $B$.) We randomly generated $N_{O}$ and $N_{T}$ pairs of objects, each with $I+R$ variables. Finally, we labeled the preferred objects according to $\mathcal{L}$.

Figure 1a shows the average performance of $G, M V$ with two different sample sizes and $V V$ for $R=15, I=0$, and $N_{T}=20$, as $N_{O}$ ranges from 2 to 20 . Figure $1 \mathrm{~b}$ shows the worst performance for each algorithm. In these figures, the data points are averages over 20 different pairs of training and test sets $(O, T)$. The average performance of $V V$ and $M V$ is better than the average performance of $G$, and the difference is significant at every data point. Also, note that the worst case performance of $G$ after seeing two observations is around 0.3 , which suggests a very poor approximation of the target. $V V$ and $M V$ 's worst case performances are much better than the worst case performance of $G$, justifying the additional complexity of the algorithms $M V$ 
and $V V$. We have observed the same behavior for other values of $R$ and $I$, and have also witnessed a significant performance advantage for $M V$ over $V V$ in the presence of irrelevant variables when training data is scarce. Space limitations prevent us from presenting these results.

Figure 2 shows the positive effect of learning bias on the performance of voting algorithms for $R=10, I=0$, and $N_{T}=20$, as $N_{O}$ ranges from 2 to 20 . In addition, this experiment aims to show that bias does not undermine the advantage voting algorithms held over the greedy algorithm in the unbiased case. To this end we have trivially generalized $G$ to produce LPMs that are consistent with a given bias. The data points are averages over 20 different pairs of training and test sets $(O, T)$. We have arbitrarily picked two biases: $B_{1}$ : $\left\{X_{1}, X_{2}, X_{3}, X_{4}, X_{5}\right\}<\left\{X_{6}, X_{7}, X_{8}, X_{9}, X_{10}\right\}$ and $B_{2}:\left\{X_{1}, X_{2}, X_{3}\right\}<\left\{X_{4}, X_{5}\right\}<\left\{X_{6}, X_{7}, X_{8}\right\}<$ $\left\{X_{9}, X_{10}\right\}$. The performance of $V V$ improved greatly with the introduction of learning biases. $B_{2}$ is a stronger bias than $B_{1}$ and prunes the space of consistent LPMs more than $B_{1}$. As a result, the performance gain due to $B_{2}$ is greater than that due to $B_{1}$. The difference between the bias curves and the non-bias curve is statistically significant except at the last point. Note that the biases are particularly effective when the number of training observations is small. The worst case performance of $G$ with biases $B_{1}$ and $B_{2}$ are also shown in Figure 2. For both biases, the worst case performance of $G$ is significantly lower than the performance of $V V$ with the corresponding bias. We obtained very similar results with $M V$ but due to space constraints we can not include them in this paper.

\section{Related Work}

Lexicographic orders and other preference models have been utilized in several research areas, including multicriteria optimization (Bertsekas \& Tsitsiklis, 1997), linear programming , and game theory (Quesada, 2003). The lexicographic model and its applications were surveyed by Fishburn (1974). The most relevant existing work for learning and/or approximating LPMs is by Schmitt and Martignon (2006) and Dombi et al. (2007), which were summarized in Section 2. Another analogy, described by Schmitt and Martignon (2006), is between LPMs and decision lists (Rivest, 1987). Specifically, it was shown that LPMs are a special case of 2-decision lists, and that the algorithms for learning these two classes of models are not directly applicable to each other.

\section{Conclusions and Future Work}

In this paper, we presented democratic approximation methods for learning a lexicographic preference model (LPM) given a set of preference observations. Instead of committing to just one of the consistent LPMs, we main-

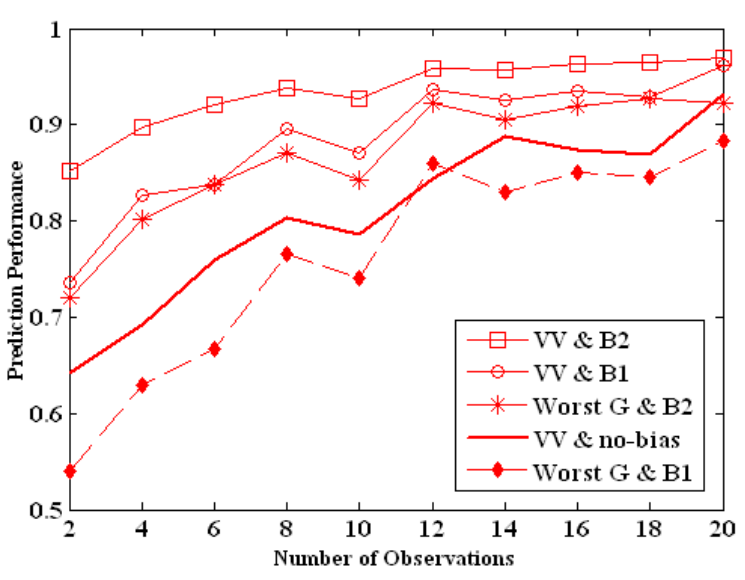

Figure 2. The effect of bias on VV and G.

tain a set of models and predict based on the majority of votes. We described two such methods: variable voting and model voting. We showed that both methods can be implemented in polynomial time and exhibit much better worstand average-case performance than the existing methods. Finally, we have defined a learning bias that can improve performance when the number of observations is small and incorporated this bias into the voting-based methods, significantly improving their empirical performance.

The future directions of this work are twofold. First, we plan to generalize our algorithms to learn the preferred values of a variable as well as the total order on the variables. Second, we intend to develop democratic approximation techniques for other kinds of preference models.

\section{Acknowledgments}

This work was supported by the Defense Advanced Research Projects Agency and the U. S. Air Force through BBN Technologies Corp. under contract number FA8650-06-C-7606.

\section{References}

Bertsekas, D., \& Tsitsiklis, J. (1997). Parallel and distributed computation: numerical methods. Athena Scientific.

Dombi, J., Imreh, C., \& Vincze, N. (2007). Learning lexicographic orders. European Journal of Operational Research, 183, 748-756.

Fishburn, P. (1974). Lexicographic Orders, Utilities and Decision Rules: A Survey. Management Science, 20, 1442-1471.

Quesada, A. (2003). Negative results in the theory of games with lexicographic utilities. Economics Bulletin, 3, 1-7.

Rivest, R. (1987). Learning decision lists. Machine Learning, 2, 229-246.

Schmitt, M., \& Martignon, L. (2006). On the complexity of learning lexicographic strategies. Journal of Machine Learning Research, 7, 55-83. 